\title{
Precision of Needle Tip Localization Using a Receiver in the Needle
}

\author{
Nikolov, Svetoslav Ivanov; Jensen, Jørgen Arendt
}

Published in:

Proceedings of 2008 IEEE International Ultrasonics Symposium

Link to article, DOI:

10.1109/ULTSYM.2008.0117

Publication date:

2008

Document Version

Early version, also known as pre-print

Link back to DTU Orbit

Citation (APA):

Nikolov, S. I., \& Jensen, J. A. (2008). Precision of Needle Tip Localization Using a Receiver in the Needle. In Proceedings of 2008 IEEE International Ultrasonics Symposium (pp. 479-482). IEEE.

https://doi.org/10.1109/ULTSYM.2008.0117

\section{General rights}

Copyright and moral rights for the publications made accessible in the public portal are retained by the authors and/or other copyright owners and it is a condition of accessing publications that users recognise and abide by the legal requirements associated with these rights.

- Users may download and print one copy of any publication from the public portal for the purpose of private study or research.

- You may not further distribute the material or use it for any profit-making activity or commercial gain

- You may freely distribute the URL identifying the publication in the public portal 


\title{
Precision of Needle Tip Localization Using a Receiver in the Needle
}

\author{
Svetoslav Ivanov Nikolov ${ }^{1,2}$ and Jørgen Arendt Jensen ${ }^{2}$, \\ 1) B-K Medical A/S, Mileparken 34, 2730 Herlev, Denmark \\ 2) Technical University of Denmark, 2800 Kgs. Lyngby, Denmark. \\ (E-mail: nikolov.svetoslav@gmail.com,jaj@elektro.dtu.dk)
}

\begin{abstract}
Many medical procedures require the detection, tracking and guidance of (biopsy) needles. The detection of the position of the needle can be challenging because of specular reflection which deflects the sound in a direction away from the transducer surface. To visualize the tip of small needles often motion is introduced to the discomfort of the patient.

Vilkomerson and co-workers suggested in 1981 the placement of an ultrasound receiver close to the needle tip. The received echoes are detected by add-on hardware. The maximum echo is assumed to originate from a beam directly above the detector, and the time of flight determines the distance to the transducer. The feasibility of the method was demonstrated by the same group in-vivo. The precision of the method has not been previously discussed in literature.

This paper introduces two methods for estimation of the position of the needle tip and investigates their precision. The first method uses conventional imaging. Instead of detecting the maximum echo, as previously suggested, the center of mass is found both across beams and along the received signals, thus decreasing the sensitivity to noise. The second method is based synthetic aperture (SA) scanning. The position of the tip is found via triangulation which involves solving a system of linear equations. The robustness to noise is ensured through averaging a number of estimates. The sensor is a ring of piezo-electric film making it possible to receive waves from any direction.

The results were obtained using simulations in Field II. The center frequency is $7 \mathrm{MHz}$. The transducer array is mechanically focused in elevation plane at $25 \mathrm{~mm}$ while the height of the elements is $4.5 \mathrm{~mm}$. The transducer pitch is 202 microns. The sensor is a ring with height of $1 \mathrm{~mm}$ and $2 \mathrm{~mm}$ diameter. Positions were varied from 10 to $120 \mathrm{~mm}$ in depth and from 0 to $20 \mathrm{~mm}$ in lateral and elevation direction. The mean error of position estimation is for the case of conventional and $\mathrm{SA}$ imaging is 0.2 and $0.05 \mathrm{~mm}$, respectively.

The precision of two methods to determine the position of the needle tip is investigated. Using spherical transmissions yields higher accuracy. Both methods can be extended to 3D if the transmissions originate from transducer elements that are not placed on a line. Needles equipped with receivers can be used for deploying brachytherapy seeds ensuring high precision of the procedure.
\end{abstract}

\section{INTRODUCTION}

Many medical procedures require the detection, tracking, and guiding the insertion of (biopsy) needles as in the case of taking biopsy samples or to deploying brachytherapy seeds. The detection of the needle position is done by using an imaging system such as CT, MRI or ultrasound scanning. The use of ultrasound-guided biopsy is widespread due to the availability and convenience of use of ultrasound scanners [2]. There are certain impediments to tracking the position of the

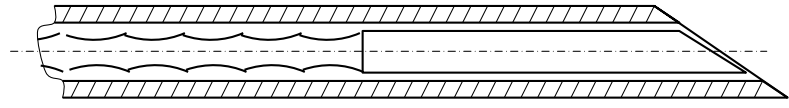

Fig. 1. Partial sectional view of a distal tip of a medical needle, illustrating echogenicity enhancement comprising of a plurality of adjacent shallow concave annular grooves about an outer surface of the stylet.

needle. Present systems use linear arrays and the position of the needle tip cannot be detected when it is outside the imaging plane. This problem has been addressed by Lee et al. by using 3D ultrasound scanning [5]. Another major problem is that the returned by the needle echo is a result of specular reflection. Often the needle is tilted at an angle relative to the ultrasound probe and remains invisible.

This has been addressed by the work of Zhao and coworkers [6] who suggest to adaptively tilt the ultrasound beam so that the incident angle is as close to $90^{\circ}$ as possible. This method enables the visualization of a part of the needle, but not necessarily the needle tip. Powers [2] suggests the generation of vibrations close at the tip of the needle, which can be detected using Doppler ultrasound.

As described above, the major problem is the smooth needle surface which gives rise to specular reflection. To increase the visibility of needles, often their surfaces are roughened in daily practice. A similar approach is suggested by Davis and McLellan [7], which suggest enhancement of the echogenicity of a needle by using a stylet with a series of annular grooves around the stylet as shown in Fig. 1.

All methods rely on echoes reflected by the needle and received by the imaging system, which identifies and visualizes the needle. This work is based on placing a receiving element or an array of receiving elements in the needle itself [8]. They will receive the pulse sent by the imaging system and the created signal will be sent for processing. Using the knowledge from the transmission and detecting the time of arrival of the pulse at the receive element in the needle, the position of that element (or elements) is calculated with high precision.

Figure 2 shows a the principle of detecting the needle. A receiver is placed near the tip of the needle. The scanner scans the region of interest using either linear, phased or synthetic aperture scanning. When the probing pulse is detected by the receiver in the needle, a signal is sent back to the scanner. From the geometry of the transmitted beam and the arrival 


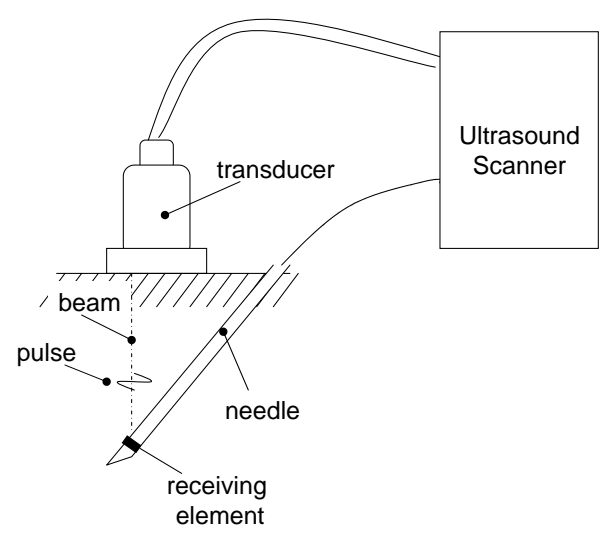

Fig. 2. Block diagram of one realization the proposed system. It consists of a scanner, transducer (single element, or array) and a needle with a receiver placed near the tip.

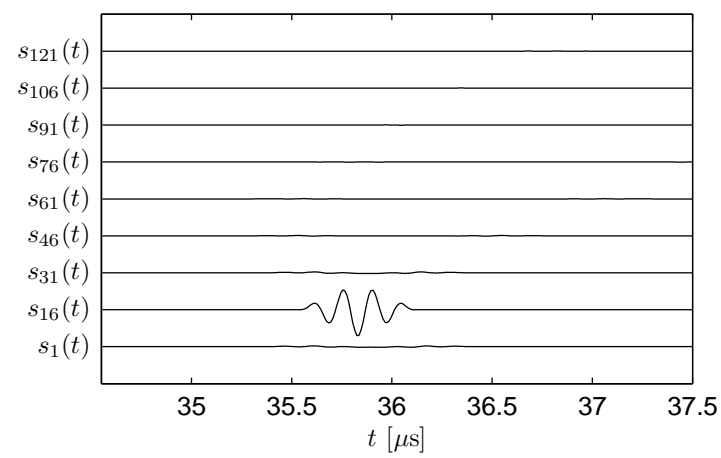

Fig. 3. Illustration of the signals received by the element placed in the needle tip.

time of the pulse, the exact position of the receive element is calculated. If two receive elements are placed in the needle, then the needle orientation is also found. The transmissions can be focused or unfocused as in the case of synthetic aperture imaging. The imaging can be in two or three spatial dimensions. The different transmit beams lead to different calculation procedures, but they are all dependent on the placement of a receive element in the needle.

\section{Calculation of the position of the needle}

\section{A. Detection of position using focused beams}

When focused beams are used, one can assume that the transmitted ultrasound pulse propagates along a line. Let the signal received by element in the needle be $s(t)$. If the element position is within the boundaries of the transmitted beam, then the received signal $s_{i}(t)$ at emission $i$ will be approximately a time-delayed and scaled version of the transmitted pulse $g(t)$ :

$$
s_{i}(t)=g\left(t-t_{p}\right),
$$

where $t_{p}$ is the time it takes for the pulse to propagate from the origin of the beam to the receiving element placed in the needle.

Figure 3 illustrates the signals received by the element placed in the needle. The indexes denote the emission (or

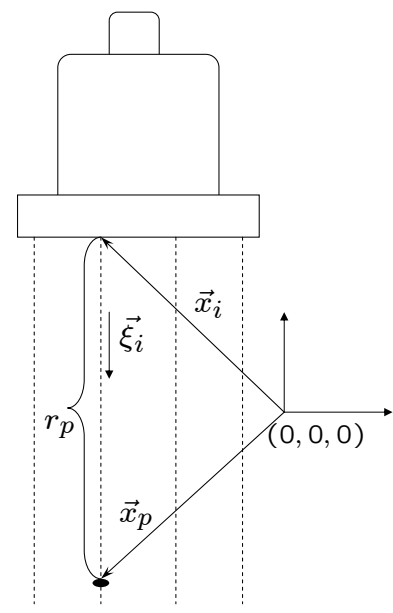

Fig. 4. Geometry used to calculate the position of the receiving element for the case of focused transmissions.

beam) number. It can be seen, that for the depicted case the maximum echo is located in the signal $s_{16}(t)$.

Figure 4 shows the geometry used to find the position of the receive element placed in the needle for the case of focused transmissions. In the figure $\vec{x}_{i}$ denotes the origin of the $i$ th transmission, for which $s_{i}(t)$ has the maximum received value, $\vec{x}_{p}$ is the position of the the receiving element, and $\vec{\xi}_{i}$ is the directional vector of the beam. The distance $r_{p}$ from the beam origin to receiving element is related to the time of arrival $t_{p}$ as

$$
r_{p}=c t_{p},
$$

where $c$ is the speed of sound. The position of the receiving element is found as

$$
\vec{x}_{p}=\vec{x}_{i}+r_{p} \vec{\xi}_{i} .
$$

The time $t_{p}$ can be estimated in a number of ways such as detecting the instance of the maximum envelope of $s(t)$ or finding the mean arrival time such as

$$
\hat{t}_{p}=\frac{\int t s^{2}(t) d t}{\int s^{2}(t) d t}
$$

The created ultrasound beams are not infinitesimally narrow and they often overlap in order to meet the spatial sampling criterion. Hence, echoes are present in several adjacent beams. A practical realization of the decision from which beam $i$ to estimate $\hat{t}_{p}$ involves for example finding the center of mass of the echoes across several signals $s_{i}(t)$ :

$$
\hat{i}(t)=\frac{\sum_{i} i s_{i}^{2}(t)}{\sum_{i} s_{i}^{2}(t)}
$$

The procedure can be united in one single search for the 2D center of mass.

The localization procedure using focused beams was described in a single plane, but its extension to 3D is straightforward. One needs only a system capable of focusing in both 


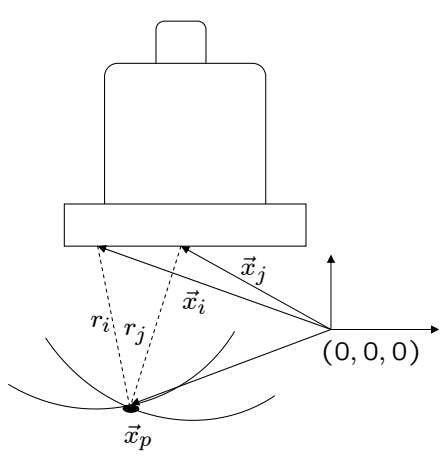

Fig. 5. Geometry used for the calculation of the position of the needle tip using spherical/circular waves.

azimuth and elevation planes.

\section{B. Detection of position using unfocused transmissions}

Unfocused transmissions can also be used to determine the position of the needle tip. Two types of waves are used plane and spherical or cylindrical/cirular waves. Plane waves can also be used. The principle is to use a transmission that creates a wave with a known wavefront. Figure 5 shows the geometry when spherical waves are transmitted. A signal $s_{i}(t)$ is received at every transmission with an origin $\vec{x}_{i}$. The time instance when the emitted pulse $g(t)$ arrives at the position of the sensor $\vec{x}_{p}$ (placed on the needle) is $t_{i}$. The distance from the origin of the spherical wave to the sensor is directly proportional to the arrival time

$$
r_{i}=t_{i} c,
$$

where $c$ is the speed of sound. Let the arrival time at emission from position $\vec{x}_{j}$ be $t_{j}$. Then the position of the sensor $\vec{x}_{p}$ lies on the cross-section of two spheres:

$$
\begin{aligned}
\left|\vec{x}_{p}-\vec{x}_{j}\right| & =r_{j}=t_{j} c \\
\left|\vec{x}_{p}-\vec{x}_{i}\right| & =r_{i}=t_{i} c
\end{aligned}
$$

To determine the position $\vec{x}_{p}$ in three dimensions, one needs at least a system of three equations. The number of emissions that can be used to localize $\vec{x}_{p}$ is limited by the number of available transducer elements, which typically is larger than 32. To get a better estimate, one can average the solutions of $\vec{x}_{p}$.

\section{RESULTS}

This section presents results from two cases: (1) the use of conventional linear-array imaging to detect the position of a sensor in 2D, and (2) the use of cylindrical waves.

In both cases scanning is performed using a linear array with 192 elements and a center frequency $f_{0}$ of $7 \mathrm{MHz}$. The transducer probe is mechanically focused in the elevation plane at a depth of $25 \mathrm{~mm}$, while the height of the elements is 4.5 $\mathrm{mm}$. The distance between the centers of 2 adjacent elements is $202 \mu \mathrm{m}$.

To demonstrate the precision of methods the signals received by point receivers were calculated. The positions of

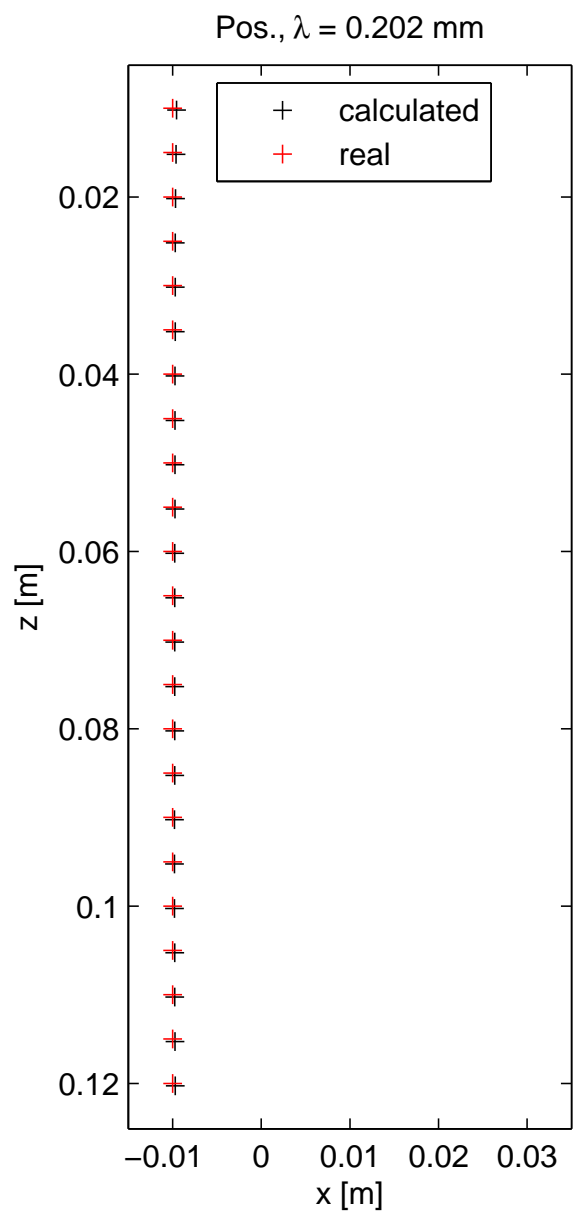

Fig. 6. Real and estimated positions of a point receiver.

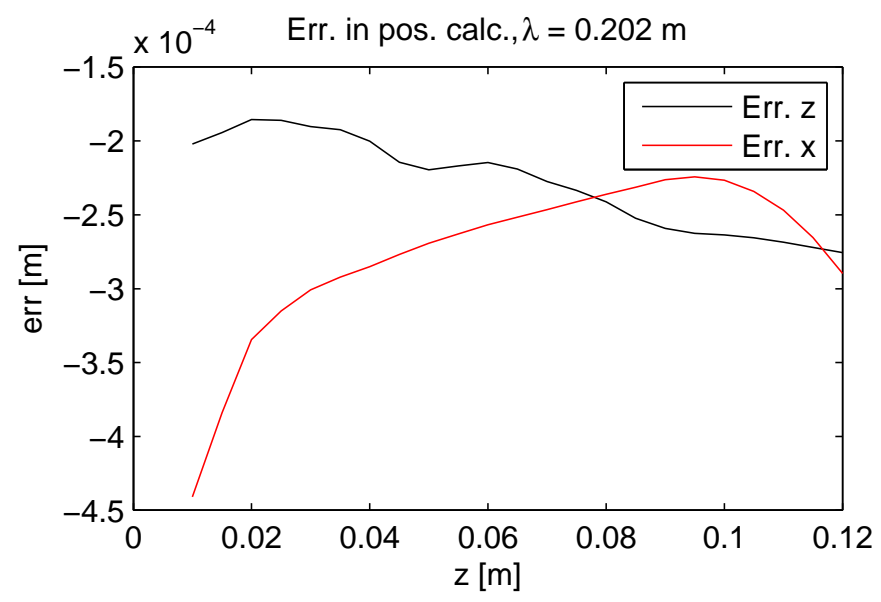

Fig. 7. Error in estimation of positions of a point receiver.

the receivers are uniformly distributed from a depth of 10 to a depth of $120 \mathrm{~mm}$ at every $5 \mathrm{~mm}$. The points are placed directly below the transducer in elevation directions and at 10 $\mathrm{mm}$ away from the middle axis of the transducer. The origin of the coordinate system is at the center of the transducer surface. 


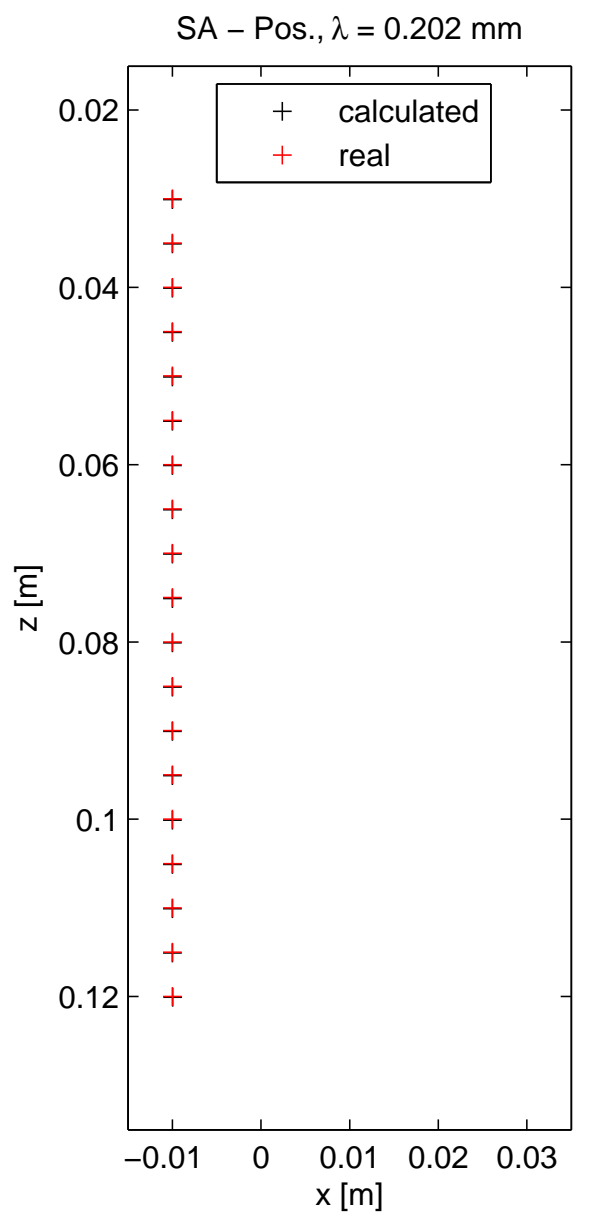

Fig. 8. Real and estimated positions of a point receiver.

\section{A. Focused transmissions}

Figure 6 shows the positions of a point receiver versus the estimated positions. Figure 7 shows the error in position estimation. The error is a fraction of a millimeter, and is comparable to one wavelength.

\section{B. Unfocused transmissions}

Figures 8 and 9 show the estimated positions and estimation errors when unfocused emissions are used. In this case only the coordinates in the azimuth plane have been estimated. The origin of the coordinate system is placed at the center of the array. Hence the coordinates $x_{p}, z_{p}$ lie on the cross-sections of two circles with origins $\vec{x}_{i}=\left(x_{i}, 0,0\right)$ and $\vec{x}_{j}=\left(x_{j}, 0,0\right)$ and radiuses $r_{i}$ and $r_{j}$, respectively. Hence, the set of equations is:

$$
\begin{array}{r}
x_{p}^{2}-2 x_{i} x_{p}+x_{i}^{2}+z_{p}^{2}=r_{i}^{2} \\
x_{p}^{2}-2 x_{j} x_{p}+x_{j}^{2}+z_{p}^{2}=r_{j}^{2},
\end{array}
$$

where $z_{p}$ is the depth of the receiver, $i$ and $j$ are the indexes of transmit elements, and $r_{i}$ and $r_{j}$ are the radiuses of the two circles respectively. $r_{i}$ and $r_{j}$ are found from the estimated arrival times $t_{i}$ and $t_{j}$. Subtracting the second from the first

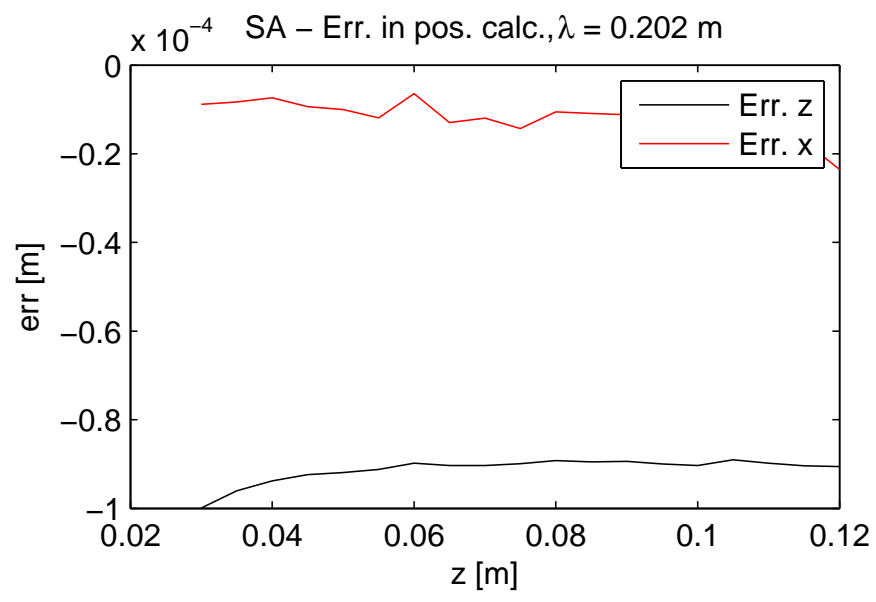

Fig. 9. Error in estimation of positions of a point receiver.

equations gives

$$
x_{p}=\frac{r_{i}^{2}-r_{j}^{2}+x_{j}^{2}-x_{i}^{2}}{2\left(x_{j}-x_{i}\right)}
$$

A total of 128 emissions have been used. $x_{p}$ was estimated 64 times, by varying $i$ and $j$ across the array $(i \in[1,64]$, and $j \in[65,128])$. The shown estimates is the mean estimate of both $x_{p}$ and $z_{p}$.

The precision of the estimates is on the order of a $\mu \mathrm{m}$, far exceeding present methods.

\section{REFERENCES}

[1] R. A. Terwilliger, "Biopsy attachment for ultrasound probe, Patent, US 4,911,173," Mar 1990, (Nov.13, 1987).

[2] J. E. Powers, "Ultrasonic imaging of biopsy needle, Patent, US 5,095,910," Mar 1992, (Apr. 18, 1990).

[3] C. Jacobs, "Brachytherapy seed needle with window, Patent ,US 6,402,677 B1,' Jun 2002, (Dec. 17, 1999).

[4] T. E. Tynes, B. Kummerlen, J. Breitenborn, H. Jurgens, S. Kohle, and J. Brandt, "Method and apparatus for positioning a biopsy needle, Patent US 2006/005263 A1,", Mar 2006, (Aug. 8, 2005).

[5] K.-J. Lee, M.-H. Bae, S.-B. Gye, J.-S-Hwang, Y.-S. Song, and G.-D. Kim, "Method and apparatus for enabling a biopsy needle to be observed, Patent, US 2003/0135119 A1," 2003, (Dec. 30, 2002).

[6] D. Zhao and Z. Y. T. E. Cupples, "Method and system for ultrasound imaging of a biopsy needle, Patent, US 2002/0173719 A1," Nov 2002, (May 15, 2001).

[7] R. E. Davis and G. L. McLellan, "Ultrasound biopsy needle, Patent, US 5,490,521," Feb 1996, (Aug. 16, 1994).

S. I. Nikolov, J. A. Jensen, and B. G. Tomov. Parametric directional beamformer. In Proc. SPIE - Progress in biomedical optics and imaging, volume 6147-13, pages 1-12, 2006

[8] D. Vilkomerson and D. Lyons, A System for Ultrasonic Beacon-Guidance of Catheters and Other Minimally-Invasive Medical Devices. IEEE Trans. Ultrason., Ferroelec., Freq. Contr., 44(2):496-504, 1997 